\title{
FAST-E en Pacientes con Trauma Abdominal Cerrado Estable, en un Departamento de Urgencias en Colombia
}

\author{
${ }^{1}$ Diego Alejandro Vivas-Giraldo, ${ }^{2}$ Gerardo Linares-Mendoza, ${ }^{3}$ Norberto Navarrete-Aldana \\ ${ }^{4}$ Carlos Hernan Carmargo-Mila, ${ }^{5}$ Karen Stephany-Perdomo, ${ }^{6}$ Luis Arcadio Cortés-Puentes
}

\section{RESUMEN}

El impacto que ha generado el trauma en Colombia a lo largo de la historia, nos ha obligado a mejorar y adaptar diferentes tipos de sistemas de atención en trauma, basados en los lineamientos internacionales, los cuales buscan evitar el significativo aumento en las tasas de mortalidad y discapacidad que se obtienen de este, especialmente en los servicios de Emergencias en los cuales se reciben el $100 \%$ de estos pacientes con traumatismo múltiple o politraumatismo, a quienes se les debe dar un abordaje rápido, efectivo y eficaz que beneficie tanto al propio paciente, como al mismo sistema de salud.

Dentro de este grupo de pacientes hay un subgrupo que son las pacientes con trauma de abdomen que cursan con estabilidad hemodinámica y además son clasificados de bajo riesgo, ya sea por índices de trauma o por otros métodos como la medición sérica de lactato, los cuales tienen un papel poco despreciable al momento de ver mortalidad y discapacidad por trauma, ya sea penetrante o cerrado; en este trabajo específicamente nos centramos en las personas que consultan al servicio de Emergencias con trauma cerrado de abdomen los cuales son considerados de bajo riesgo, siendo este subgrupo de pacientes uno de los mas difíciles de abordar y enfocar al momento de la valoración inicial, ya que se debe tener la

\footnotetext{
${ }^{1}$ Residente de Medicina de Emergencias, ${ }^{2,6}$ Especialista en Medicina de Emergencias, ${ }^{3}$ Especialista en Medicina de Emergencias, ${ }^{4}$ Especialista en Cirugía General, ${ }^{5}$ Médico Interno

${ }^{1}$ Universidad del Rosario/Fundación Santa fe de Bogotá Especialista en Gerencia Hospitalaria-ESAP

${ }^{2,6}$ Fundación Cardioinfantil/Instituto de Cardiología, Brazil

${ }^{3}$ Universidad del Rosario, Candidato a Maestria en Epidemiología Clínica-Universidad Javeriana, Emergenciologo Unidad de Cuidado Intensivo de Quemados-Hospital Simon Bolivar Colombia

${ }^{4}$ Jefe Departamento de Urgencias Adultos-Fundación Cardioinfantil/Instituto de Cardiología, Brazil

${ }^{5}$ Universidad de la Sabana/Fundación Cardioinfantil/Instituto de Cardiología, Brazil
}

Corresponding Author: Diego Alejandro Vivas-Giraldo Residente de Medicina de Emergencias-Universidad del Rosario/Fundación Santa fe de Bogotá, Especialista en Gerencia Hospitalaria_ESAP, e-mail: alejandro_048@ hotmail.com seguridad de que no hay lesiones que comprometen la vida y por consiguiente estos pacientes puedan ser dados de alta.

Una de las practicas que mas se han venido haciendo en los últimos años en la valoración del paciente con trauma de abdomen cerrado es la ultrasonografía (FAST), la cual ha logrado disminuir los tiempos en la toma de decisiones, además de disminuir tasa de complicaciones en estos pacientes; con base en esto se ha implementado en la Fundación Cardioinfantil este procedimiento en el servicio de Emergencias, encabezado por el grupo de ultrasonido a la cabecera del paciente (Emergenciólogos), con lo cual se busca medir el impacto del trauma cerrado de abdomen clasficado de alta energia y de bajo riesgo.

Palavras chave: FAST-E, Trauma abdominal cerrado estable.

How to cite this article: Vivas-Giraldo DA, Linares-Mendoza G, Navarrete-Aldana N, Carmargo-Mila $\mathrm{CH}$, Stephany-Perdomo K, Cortés-Puentes LA. FAST-E en Pacientes con Trauma Abdominal Cerrado Estable, en un Departamento de Urgencias en Colombia. Panam J Trauma Crit Care Emerg Surg 2015;4(2):59-65.

Source of support: Nil

Conflict of interest: None

\section{ABSTRACT}

To improve and adapt different types of trauma care systems, based on international guidelines and to avoid significant increase in mortality and disability, we focus on a subgroup of patients who are abdominal trauma patients that present with hemodynamic stability. In recent years abdominal ultrasonography (FAST has managed to reduce time in decision-making, and reduce complication rate in these patients. In our observational study of 65 patients with high mechanism blunt thoracoabdominal trauma and negative EFAST, only $3 \%$ required further investigation after an observational period with CT scan.

Keywords: Blunt abdominal trauma, FAST.

\section{INTRODUCCIÓN}

A lo largo del tiempo la consulta de urgencias por trauma ha sido una de las situaciones mas comunes y complejas de manejar en los diferentes hospitales a nivel mundial, permitiendo con este, forjar la creación de diferentes tipos de protocolos, guías y formas de proceder ante este tipo de pacientes, los cuales demandan atención inmediata y,

Note: Paper presented in Resident Research Competition at the annual congress of Panamerican Trauma Society, Panama City, Panama, November 2015. 
eficaz y eficiente por parte del personal de los servicios de urgencias, evitando al máximo la presentación de eventos adversos y complicaciones que deriven en daño a los pacientes. ${ }^{1}$

Entre este gran número de consultas se encuentran los pacientes con trauma múltiple, el cual se define como la lesión de una o más regiones corporales (AIS no mayor a ${ }^{3,1}$ sin signos de respuesta inflamatoria sistémica, siendo estos por sus características tanto anatómicas como fisiológicas los mas comúnmente recibidos en las instituciones de salud; ${ }^{1}$ igualmente es de gran importancia el abordaje de este tipo de trauma ya que existe una alta posibilidad de tener trauma de abdomen cerrado cuando hay trauma múltiple con cinemática de trauma de alta energía, ya sea por colisiones directas, caídas o por ondas expansivas que lesionan el abdomen. ${ }^{1}$

La cinemática del trauma es muy importante a la hora de abordar pacientes con trauma múltiple ya que ayuda al examinador a sospechar las posibles lesiones asociadas al evento, para esto hay que tener clara la definición de trauma de alta energía, la cual consiste en personas que presentan eyección del vehículo involucrado en el accidente, conductor de motocicleta, que hayan muertos en la escena del accidente, que haya una caída de 6 o más metros de altura, o una caída de 5 o mas escalones, colisiones entre vehículos a maás de $55 \mathrm{~km} /$ hora, colisiones de motocicleta a más de $30 \mathrm{~km} /$ hora o colisión auto-peatón a mas de $30 \mathrm{~km} /$ hora, y colisión a un niño a cualquier velocidad. ${ }^{4}$ Teniendo en cuenta que las velocidades entre los vehículos involucrados se suman entre si; por ejemplo, si hay una colisión entre dos vehículos de los cuales uno iba a 40 $\mathrm{km} /$ hora y el otro iba a $35 \mathrm{~km} /$ hora, la energía total con que estos vehículos se estrellaron fue de $75 \mathrm{~km} /$ hora; ${ }^{4}$ igualmente se considera trauma de alta energía todo accidente en el cual la extricación de la persona afectada se demore mas de 20 minutos o que tenga un puntaje RTS (revised trauma score) menor o igual a 11 en la escena; ${ }^{4}$ de igual manera hay otros parámetros para definir trauma de alta energía que pueden ser valorados en la primera intervención al paciente los cuales son los signos vitales y la presencia de lesiones penetrantes en cabeza, cuello, tórax y abdomen, presencia de dos o mas fracturas de huesos largos, amputaciones traumáticas, trauma raquimedular y quemadura de la vía aérea o de más del $20 \%$ de la superficie corporal en adultos y el $10 \%$ en niños. ${ }^{4}$ además de lo anterior se deben tener en cuenta los grupos especiales por su mayor vulnerabilidad a la hora de presentar traumatismo múltiple, embarazo, diabetes mellitus, inmunosupresión, anticoagulación, falla cardiaca, disfunción respiratoria y edades extremas (menor 5 años y mayores 65 años), ya que estos grupos tienen un riesgo aumentado de presentar complicaciones tempranas relacionadas con el trauma. ${ }^{6}$

Los pacientes con trauma de abdomen cerrado son una entidad compleja de estudiar, debido a las incertidumbre constante de la presencia de lesiones intraabdominales y retroperitoneales que muchas veces tardan o no se manifiestan clínicamente de alguna forma que alarme al personal de urgencias para actuar e intervenirles de inmediato, ${ }^{6}$ requiriendo entonces largas observaciones en los servicios de urgencias, debido a la baja sensibilidad del examen físico, el cual se debe hacer de forma seriada para lograr un adecuada valoración clínica de pacientes con trauma cerrado de abdomen, sin embargo, esto a costa de periodos de observaciones inclusive superiores a 8 horas en algunos casos. ${ }^{6}$ Debido a lo anterior, con el paso del tiempo se han instaurado diversos protocolos de manejo de trauma de abdomen cerrado, con los que se busca básicamente localizar lesiones de forma mas rápida y determinar el riesgo de desarrollarlas durante la observación clínica de las mismas, tratando así de definir estos pacientes en menos tiempos, ${ }^{6}$ sin tener que aumentar el número de complicaciones y eventos adversos, se han utilizado protocolos de observación y tolerancia a la vía a oral, ${ }^{6}$ protocolos con realización de tomografía de abdomen con medio de contraste para descartar lesiones intraabdominales aunque con el riesgo de complicaciones por aplicación de medio de contraste y las largas horas de espera por la preparación que este estudio requiere, ${ }^{6}$ y la realización de Ultrasonografía de emergencias con FAST-E para de una forma rápida descartar presencia de líquido libre en la cavidad abdominal. ${ }^{7}$

En la actualidad, el trauma es la principal causa de muerte dentro de las primeras cuatro décadas de la vida. ${ }^{7}$ El trauma abdominal aislado o asociado con otros tipos de trauma presentan un importante reto para el medico que atiende urgencias. El traumatismo cerrado de abdomen, se pueden asociar a mayor mortalidad por la dificultad en el diagnostico o al ser los síntomas enmascarados o pasar desapercibidos por otros tipos de trauma. ${ }^{9}$

Los diagnósticos erróneos o tardíos son el principal riesgo en morbilidad y mortalidad en este tipo de lesiones por lo cual deben ser evaluadas y descartadas en forma organizada y sistemática por un equipo de trauma. ${ }^{9}$ El conocimiento del mecanismo de trauma permite anticipar lesiones ocultas, dirigir las pruebas diagnosticas e iniciar tratamientos en forma mas rápida. ${ }^{9}$ Mecanismos de alta energía han sido descritos, asociados a mayor probabilidad de lesiones ocultas, incluidas las abdominales, con mayor riesgo de mortalidad, que requieren estudios diagnósticos y manejo especializado en centros de trauma. ${ }^{9}$

El manejo del trauma abdominal cerrado con inestabilidad hemodinámica ha sido modificado 
positivamente gracias a la valoración ultrasonográfica enfocada al trauma (FAST-E, por sus siglas en inglés), con alta sensibilidad (86\%) y especificidad $(99 \%),{ }_{1}^{9}$ para la detección de líquido libre intraabdominal, secundario a lesiones significativas que requieren manejo quirúrgico. Sin embargo, en el manejo del trauma abdominal cerrado con paciente estable, la utilidad del FAST-E ha sido cuestionada por trabajos que determinaron baja sensibilidad (45\%) y especificidad $(94 \%),{ }^{7,9}$ que impiden tomar decisiones o plantear manejo quirúrgico. Por lo anteriormente mencionado, se han descrito protocolos que involucran observación prolongada del paciente basados en examen físico seriado y/o evaluación con tomografía abdominal total. ${ }^{9}$ Recientemente se han descrito estudios de trauma abdominal cerrado en pacientes estables, basados en FAST-E y examen físico normal, con resultados prometedores en pacientes de bajo riesgo. ${ }^{9}$ Nuestra institución adoptó un protocolo para pacientes estables, con sospecha de trauma abdominal debido al mecanismo peligroso de trauma, basado en FAST-E y examen físico normal sumado a lactato menor a $2.5 \mathrm{mmol} / 1$, como marcador de bajo riesgo, con el cual se busca describir el número de lesiones intra abdominales, re consultas y tiempo promedio de observación en el servicio de urgencias de este grupo de pacientes incluidos en este protocolo de estudio.

\section{OBJETIVO GENERAL}

Describir los resultados de la práctica clínica actual para el estudio de pacientes adultos con sospecha de trauma abdominal cerrado debido a un mecanismo de trauma de alta energía, hemodinamicamente estable en el Departamento de Emergencias de un hospital de cuarto nivel de complejidad en Bogotá-Colombia.

\section{OBJETIVOS ESPECIFICOS}

1.1 Describir los mecanismo de trauma más frecuentemente relacionados con sospecha de trauma cerrado de abdomen con estabilidad hemodinámica.

1.2 Determinar el número de re consultas relacionas por lesiones abdominales no diagnosticada y otras causas posterior al egreso hospitalario de los pacientes valorados con el protocolo de FAST-E y lactato.

1.3 Describir el tiempo promedio de observación de los pacientes valorados con el protocolo de FAST-E y lactato.

\section{METODOLOGÍA}

Estudio observacional descriptivo, con cohorte histórica. Se analizan los pacientes que ingresaron al Departamento de Emergencias de un hospital de cuarto nivel de complejidad en Bogotá-Colombia, con trauma múltiple y mecanismo peligroso de alta energía, durante el periodo comprendido desde el 1 de julio de 2013 al 28 de febrero de 2014. Estos pacientes cumplieron los siguientes criterios: ser mayores de 17 años, tener mecanismo de trauma cerrado, estabilidad hemodinámica, lactato sérico menor a 2,5 mmol/L, examen físico sin alteraciones anatómicas abdominales y poseer un RTS mayor a 11. En la práctica clínica institucional los pacientes con los anteriores criterios fueron valorados con un FAST-E. Si el examen no demuestra líquido libre intraabdominal, se clasifico como de baja probabilidad para tener lesiones intraabdominales y no se solicitaron estudios adicionales. Aquellos pacientes en quienes por el mecanismo del trauma y/o el criterio del tratante, se hizo necesario la realización de una tomografía abdominal, fueron excluidos. Se registraron las características demográficas, las lesiones asociadas, el tiempo de observación abdominal, el resultado final durante la observación y si se presento reingresos. En pacientes que requirieron hospitalización por otras causas, se reviso la historia clínica con el fin de determinar si se encontró alguna lesión abdominal no diagnosticada inicialmente.

Se realizará una revisión de la base de datos de ingresos primarios asociado a trauma al servicio de Urgencias de FCI. durante durante el periodo comprendido entre el 1 de Julio de 2013 y el 28 de Febrero de 2014. estableciendo total de traslados primarios. Y se eliminaron pacientes con trauma penetrante, Posteriormente se evaluó la gasimetría arterial y los criterios de inclusión. Posteriormente se registran las variables en estudio en un archivo plano de excel.

Se hizo seguimeinto telefónico para verifcar si el paciente requirió valoración por Urgencias u hospitalización por causa abdominal y/o otras causas, y si los hallazgos son relevantes (Cirugía abdominal, necesidad de transfusión, necesidad de TAC abdominal) (Se revisará la historia clínica con el fin de determinar si se encontró alguna lesión abdominal no diagnosticada inicialmente).

Se realizará un análisis descriptivo de las variables en estudio. Para las variables categóricas se realizará distribución de frecuencias (porcentajes). Para las variables continuas se realizara medidas de tendencia central y dispersión (media y desviación estándar).

\section{IMPACTO}

Con la realización de este trabajo se busca determinar si con la descripción del uso de protocolo de FAST-E en pacientes con trauma de abdomen cerrado con mecanismo peligroso y con bajo riesgo medido por lactato sérico, se pueda disminuir la toma de imagenes 
innecesarias (Tomografía de Abdomen), generando de esta manera disminución en el tiempo de observación de este grupo de pacientes, logrando así plantear nuevas hipotesis para realizar investigaciones a futuro.

\section{RESULTADOS}

Se evaluaron un total de 114 pacientes con sospecha de trauma abdominal a quienes se les realizó FAST-E, de los cuales se descartaron 8 por ser trauma penetrante, 6 por inestabilidad hemodinámica y 35 por tener alteraciones en el examen físico y/o gases arteriales que requirieron TAC abdominal. Un total de 65 pacientes cumplieron los criterios de inclusión de los cuales la mayoría fueron traumas por atropellamiento de peatón y/o bicicleta $(29,2 \%)$ y traumas en motocicleta ( $21,5 \%)$, seguidos por colisiones a alta velocidad $(13,8 \%)$. El valor promedio de lactato de estos pacientes fue de 1,49 y se requirió en promedio 7,7 horas de observación para que fueran clasificados de baja probabilidad de tener lesiones intraabdominales. Al revisar la base de datos de estos pacientes se encontró que 10 (15,3\%) pacientes tuvieron una hospitalización mayor a 48 horas sin mención de lesiones abdominales diagnosticadas a posteriori, $18(27,6 \%)$ pacientes reingresaron por otra causa relacionadas al trauma, principalmente en extremidades sin necesidad de estudiar lesiones abdominales adicionales y 2 (3\%) pacientes reingresaron por dolor abdominal con TAC normal.

\section{DISCUSIÓN}

Los pacientes con mecanismo de trauma de alta energía, como los pacientes de nuestra descripción, a pesar de no tener criterios fisiológicos o anatómicos de severidad, pueden aún tener lesiones ocultas. El presentar un mecanismo de trauma peligroso, tienen una sensibilidad baja $(50 \%$ a $70 \%)$, con valores predictivos positivos del 16,1\%, para detectar traumas con ISS $>16$. Por lo anterior estos pacientes deben ser trasladados para ser valorados por un equipo de trauma, que utilice estrategias para descartar lesiones ocultas, incluyendo las intraabdominales. El FAST-E se considera una examen fácil de realizar, sin riesgos potenciales para el paciente, reproducible a cualquier momento y evita la necesidad de transportar al paciente fuera del Departamento de Emergencias. A pesar de esto, no ha demostrado ser una herramienta efectiva en el roll-out y/o roll-in de pacientes con trauma abdominal cerrado estable. Sin embargo, como sugiere Byars y colaboradores, podría ser llegar a comprobarse la utilidad del FAST-E en cierto tipo de pacientes de bajo riesgo. En su estudio Byars y colaboradores, reporta que aproximadamente el 9,2\% de los pacientes con examen físico normal y
FAST-E negativo, presentaban alguna alteración en la tomografía, sin configurar lesiones significativas de intervención quirúrgica o de mortalidad. En nuestra práctica clínica, nosotros agregamos el lactato menor a 2,5 como indicador de bajo riesgo y estos pacientes fueron observados por aproximadamente 8 horas, sin haber evidencia de lesiones abdominales significativas registradas en la historia clínica. Sólo se registraron 2 reingresos por sintomatología abdominal relacionada al trauma, sin embargo en ningún paciente presentó alguna lesión demostrable con tomografía.

\section{CONCLUSIÓN}

El papel del FAST-E en pacientes con trauma abdominal cerrado estables hemodinámicamente y de bajo riesgo debe replantearse. En nuestra descripción los pacientes con un mecanismo de trauma peligroso, con lactato menor a $2,5 \mathrm{mmol} / \mathrm{dl}$, examen físico normal, FAST-E negativo y observación aproximada de 8 horas, no presentaron lesiones intraabdominales que requirieran estrategias diagnosticas adicionales. Este protocolo debe ser valorado prospectivamente involucrando mayor número de pacientes con el fin de confirmar la seguridad de dicha practica clínica.

\section{REFERENCIAS}

1. Nishijima DK, Simel DL, Wisner DH, Holmes JF. Does this adult patient have a blunt intra-abdominal injury? JAMA 2012 Apr;307(14):1517-1527.

2. Simon Fleming. Accuracy of FAST scan in blunt abdominal trauma in a major London trauma centre. Int J Surg 2012; 10:470-474.

3. Schurink GW, Bode PJ, van Luijt PA, van Vugt AB. The value of physical examination in the diagnosis of patients with blunt abdominal trauma: a retrospective study. Injury 1997;28(4):261e5.

4. Sheng AY. Focused assessment with sonography in trauma and abdominal computed tomography utilization in adult trauma patients: trends over the last decade. Emerg Med Int 2013.

5. Natarajan B. FAST scan: is it worth doing in hemodynamically stable blunt trauma patients?. Surgery 2010;148:695-701.

6. FAST Enough? A Validation Study for Focused Assessment with Sonography for Trauma Ultrasounds in a Level II Trauma Center GALEN HOLMES.

7. Focused Assessment with Sonography in Trauma and Abdominal Computed Tomography Utilization in Adult Trauma Patients: Trends over the Last Decade.

8. Marx JA, Isenhour JL. Abdominal Trauma. In: Emergency Medicine Concepts and Clinical Practice. 6th ed. Marx. JA Eds. Elsevier; 2006.

9. Davis JJ, Cohn I Jr, Nance FC. Diagnosis and management of blunt abdominal trauma. Ann Surg 1976;183(6):672.

10. Gebhard F, Huber-Lang M. Polytrauma pathophysiology and management principles. Langenbecks Arch Surg 2008;393(6): 825-831.

11. Newgard CD, Lewis RJ, Kraus JF, McConnell KJ. Seat position and the risk of serious thoracoabdominal injury in lateral 
motor vehicle crashes. Accid Anal Prev 2005 Jul;37(4):668-674. Epub 2005 Apr 2.

12. Newgard CD, Lewis RJ, Jolly BT. Use of out-of-hospital variables to predict severity of injury in pediatric patients involved in motor vehicle crashes. Ann Emerg Med 2002 May;39(5):481-491.

13. Newgard CD, Lewis RJ, Kraus JF. Steering wheel deformity and serious thoracic or abdominal injury among drivers and passenger sinvolved in motor vehicle crashes. Ann Emerg Med 2005;45(1):43.

14. Brasel KJ, Nirula R. What mechanism justifies abdominal evaluation in motor vehicle crashes?. J Trauma 2005;59(5):1057.

15. Validation of a prediction rule for the identification of children with intra-abdominal injuries after blunt torso trauma. Holmes JF, Mao A, Awasthi S, McGahan JP, Wisner DH, Kuppermann N. Ann Emerg Med 2009 Oct;54(4):528-533.

16. Takishima T, Sugimoto K, Hirata M, Asari Y, Ohwada T, Kakita A. Serum amylase level on admission in the diagnosis of blunt injury to the pancreas: its significance and limitations. Ann Surg 1997;226(1):70.

17. Mofidi M, Hasani A, Kianmehr N. Determining the accuracy of base deficit in diagnosis of intra-abdominal injury in patients with blunt abdominal trauma. Am J Emerg Med 2010 Oct;28(8):933-936.

18. Charles A. Sepsis Biomarkers in Polytrauma Patients. Crit Care Clin 2011;27:345-354.

19. Martin MJ. Discordance between lactate and base deficit in the surgical intensive care unit: which one do you trust? Am J Surg 2006;191:625-630.

20. Schnüriger B, Inaba K, Barmparas G, Eberle BM, LustenbergerT, Lam L, Talving P, Demetriades D. Serial white blood cell counts in trauma: do they predict a hollow viscus injury? J Trauma 2010;69(2):302.

21. Mele TS, Stewart K, Marokus B, O'Keefe GE. Evaluation of a diagnostic protocolus in screening diagnostic peritoneal lavage with selective use of abdominal computed tomography in blunt abdominal trauma. J Trauma 1999;46(5):847.
22. Bhagvan S, Turai M, Holden A, Ng A, Civil I. Predicting hollow viscus injury in blunt abdominal trauma with computed tomography. World J Surg 2013 Jan;37(1):123-126.

23. Soto JA, Anderson SW. Multidetector CT of blunt abdominal trauma. Radiology 2012;265(3):678.

24. Holmes JF, McGahan JP, Wisner DH. Rate of intra-abdominal injury after a normal abdominal computed tomographic scan in adults withblunt trauma. Am J Emerg Med 2012 May;30(4):574-579.

25. Heilbrun ME, Chew FS, Tansavatdi KR, Tooze JA. The role of negative $\mathrm{CT}$ of the abdomen and pelvis in the decision to admit adults from the emergency department after blunt trauma. J Am Coll Radiol 2005 Nov;2(11):889-895.

26. Holmes JF, Wisner DH, McGahan JP, Mower WR, Kupperman N. Clinical prediction rules for identifying adults at very low risk for intra-abdominal injuries after blunt trauma. Ann Emerg Med 2009;54(4):575.

27. American College of Emergency Physicians. Emergency ultrasound imaging criteria compendium. American College of Emergency Physicians. Ann Emerg Med 2006;48(4): 487.

28. Blackbourne LH, Soffer D, McKenney M, Amortegui J, Schulman CI, Crookes B, Habib F, Benjamin R, Lopez PP, Namias N, Lynn M, Cohn SM. Secondary ultrasound examination increases the sensitivity of the FAST exam in blunt trauma. J Trauma 2004;57(5):934.

29. Instituto nacional de medicina legal y ciencias forenses. Boletines estadísticos mensuales. Comparativo 2010-2011 Muertes violentas según contexto; 2012 (Consultado por ultima vez Agosto de 2014). Disponible en www. medicinalegal.gov.co

30. Physical examination combined with focused assessment with sonography for trauma examination to clear hemodynamically stable blunt abdominal trauma patients. Am J Emerg Med 2013 Oct; 31(10):1527-1528.

31. Guía de Trauma abdominal penetrante. Universidad del valle. Diciembre de 2003. 


\section{Dr Vivas re : 'Fast-E En Pacientes Con Trauma Abdominal Cerrado Estable, En Un Departamento De Urgencias En Colombia'}

Dr Vivas presents an important issue. Can EFAST be enough to rule out important injuries in our blunt trauma patients? He brings us an observational study of 65 patients with high mechanism blunt thoraco-abdominal trauma and negative EFAST, of whom only $3 \%$ required further investigation after an observational period with CT scan. This is an interesting concept since this examination has been used in unstable patients to triage to the operating room; not as the sole tool to rule out injuries and discharge patients home. In places with almost unlimited resources; it seems patients tend to receive more and more radiological studies and with it radiation that might; or might not be necessary. Overtime, the numbers that indicate the vital signs in the monitors have gotten larger and larger, and sometimes the physicians hands further and further away from the patient's body. ${ }^{1-5}$

Performing serial ultrasound exams, forces the physician to touch the patient. As it was described previously by experts, such as Dr Rozycki, this is an extension of the physical exam. A visual stethoscope. I congratulate Dr Vivas for asking this difficult question, especially since in medicine we must adapt the tools to the environment we live in order to provide for the best patient care. With less resources less capacity for expensive tests. Although as an ultrasound enthusiast I would love to endorse the concept he advocates for in his paper, unfortunately; the short follow-up and the small number of patients in this study prevents me from doing so at the present time.

I will, however, encourage Dr Vivas curiosity, and add a challenge. If you add to the EFAST a cardiac view—not only to look for effusion but to evaluate fluid status and contractility, now you will also have a portable hemodynamic monitor in your hands. This might make the process of triage faster and more informed.

Congratulations on a wonderfully written paper, and I am looking forward your future research in this matter.

\section{REFERENCES}

1. Ferrada P, Anand RJ, Whelan J, Aboutanos MA, Duane T, Malhotra A, et al. Limited transthoracic echocardiogram: so easy any trauma attending can do it. J Trauma 2011;71(5):1327-1331.

2. Ferrada P, Vanguri P, Anand RJ, Whelan J, Duane T, Aboutanos M, et al. A, B, C, D, echo: limited transthoracic echocardiogram is a useful tool to guide therapy for hypotension in the trauma bay—a pilot study. J Trauma Acute Care Surg 2013;74(1):220-223.

3. Ferrada P, Evans D, Wolfe L, Anand RJ, Vanguri P, Mayglothling J, et al. Findings of a randomized controlled trial using limited transthoracic echocardiogram (LTTE) as a hemodynamic monitoring tool in the trauma bay. J Trauma Acute Care Surg 2014; 76(1):31-37.

4. Rozycki GS, Ballard RB, Feliciano DV, Schmidt JA, Pennington SD. Surgeon-performed ultrasound for the assessment of truncal injuries: lessons learned from 1540 patients. Ann Surg 1998;228(4):557-567.

5. Rozycki GS, Ochsner MG, Feliciano DV, Thomas B, Boulanger BR, Davis FE, et al. Early detection of hemoperitoneum by ultrasound examination of the right upper quadrant: a multicenter study. J Trauma 1998;45(5):878-883. 


\section{Dr Vivas re : 'Fast-E En Pacientes Con Trauma Abdominal Cerrado Estable, En Un Departamento De Urgencias En Colombia'}

Dr Vivas presenta un cuestionamiento importante. ¿Puede EFAST ser suficiente para diagnosticar lesiones importantes en nuestros pacientes con traumatismo abdominal cerrado? Él nos trae un estudio observacional de 65 pacientes con alto mecanismo contundente de trauma toraco-abdominal y examen de EFAST negativo, de los cuales sólo el 3\% requirió más investigación después de un período de observación con TAC. Este es un concepto interesante ya que este examen se ha usado en pacientes inestables como traige para la sala de operaciones; no como la única herramienta para descartar lesiones completamente. En lugares con recursos casi ilimitados; parece pacientes tienden a recibir más y más estudios radiológicos y con esto también radiación que puede; o no podría ser necesaria. Con el tiempo, los números que indican los signos vitales en los monitores han vuelto más y más grande, y en ocasiones las manos de los médicos alejándose más y más del cuerpo del paciente.

La realización de exámenes de ultrasonido de serie, obliga al médico a tocar al paciente. ${ }^{1-5}$ Como se describió previamente por expertos como la Dra. Rozycki, es una extensión del examen físico. Un estetoscopio visual. Felicito Dr Vivas por hacer esta pregunta difícil, sobre todo porque en la medicina hay que adaptar las herramientas para el medio ambiente en que vivimos con el fin de proporcionar la mejor atención al paciente. Con menos recursos también existe menor capacidad para pruebas costosas. Aunque como un entusiasta de ultrasonido me encantaría avalar el concepto que defiende en su artículo, desafortunadamente; el corto seguimiento y el pequeño número de pacientes en este estudio me impide hacerlo en el momento actual.

Sin embargo, le animaré al Dr Vivas su curiosidad, y le quiero añadir un desafío. Si se agrega a la EFAST una vista-cardiaca no sólo para buscar derrame sino para evaluar el estado del fluido y la contractilidad, ahora también tendrá un monitor hemodinámico portátil en sus manos. Esto puede hacer que el proceso de triaje más rápido y mejor informado.

Felicitaciones por un trabajo maravillosamente escrito, y estaré pendiente en leer su investigación futura en esta materia.

\section{REFERENCES}

1. Ferrada P, Anand RJ, Whelan J, Aboutanos MA, Duane T, Malhotra A, et al. Limited transthoracic echocardiogram: so easy any trauma attending can do it. J Trauma 2011;71(5):1327-1331.

2. Ferrada P, Vanguri P, Anand RJ, Whelan J, Duane T, Aboutanos M, et al. A, B, C, D, echo: limited transthoracic echocardiogram is a useful tool to guide therapy for hypotension in the trauma bay—a pilot study. J Trauma Acute Care Surg 2013;74(1):220-223.

3. Ferrada P, Evans D, Wolfe L, Anand RJ, Vanguri P, Mayglothling J, et al. Findings of a randomized controlled trial using limited transthoracic echocardiogram (LTTE) as a hemodynamic monitoring tool in the trauma bay. J Trauma Acute Care Surg 2014; 76(1):31-37.

4. Rozycki GS, Ballard RB, Feliciano DV, Schmidt JA, Pennington SD. Surgeon-performed ultrasound for the assessment of truncal injuries: lessons learned from 1540 patients. Ann Surg 1998;228(4):557-567.

5. Rozycki GS, Ochsner MG, Feliciano DV, Thomas B, Boulanger BR, Davis FE, et al. Early detection of hemoperitoneum by ultrasound examination of the right upper quadrant: a multicenter study. J Trauma 1998;45(5):878-883.

Prof Paula Ferrada

Richmond, Virginia, USA 